\title{
Moving element analysis of high-speed rail system accounting for hanging sleepers
}

\author{
Jian Dai ${ }^{1}$, Kok Keng Ang ${ }^{1, *}$, and Dongqi Jiang ${ }^{2, *}$ \\ ${ }^{1}$ Department of Civil \& Environmental Engineering, National University of Singapore, Singapore, 117576 \\ ${ }^{2}$ Department of Civil Engineering, School of Science, Nanjing University of Science and Technology, Nanjing, China, 210094
}

\begin{abstract}
It is very common in the ballasted track system that sleepers are not well supported by the ballast materials due to the uneven settlement of the ballast under repeated train passage. These unsupported track elements are often termed as hanging sleepers and they can lead to undesirable effects due to increased dynamic response of the train-track system, especially when the speed of the train is high. In this paper, we present a computation scheme in conjunction with the moving element method for the analysis of highspeed train-track dynamics accounting for hanging sleepers. The proposed computational scheme will be first verified by comparison with available analytical results. The dynamic response of a high-speed train traveling on a ballasted track considering unsupported sleepers is next investigated. Various factors affecting the response of the high-speed rail system including the speed of the train, the number of hanging sleepers and the pattern of the hanging sleepers will be examined and discussed.
\end{abstract}

\section{Introduction}

The high-speed train travel has become increasingly popular in continental people's daily life, for it does not only greatly shorten the inter-city travel time without causing traffic congestion but also being environmental friendlier as compared to the conventional car travel. The rapid increase in speed, however, also gives rise to concerns regarding the train-travel safety and increased demand on railway system maintenance. For example, the vibration of rails induced by a passing train would disturb the pavement of ballast materials [1]. According to [1], the ballast would undergo non-elastic deformation under vehicle passage which eventually leads to uneven track settlement and voids under sleepers. Literature has shown that it is very common that sleepers are not well supported by the ballast [2]. The gaps between sleepers and ballast put the safety of train operation in question, and the risk of disaster is believed to be amplified with regards to the increase in the running speed of the train.

The existence of unsupported sleepers, also known as hanging sleepers, has attracted railway engineers' attention, and quite a few research studies, both experimental and numerical, were carried out to investigate the effect of hanging sleepers on the dynamic response of a train-track system [3-5]. These works mainly assume that the hanging sleepers only appear in a consecutive arrangement. In reality, however, it is common that the sleepers are not aligned in a consecutive manner. In other words, well-bedded sleeper(s) may be surrounded by hanging sleepers. Unfortunately, such scenario has been rarely studied.

\footnotetext{
* Corresponding author: Kok Keng Ang: ceeangkk@nus.edu.sg Dongqi Jiang: jiangdongqi@gmail.com
}

Due to the complexity of the hanging sleeper problem, most researchers resorted to numerical solutions such as finite element method (FEM) and finite difference method (FDM). Although these approaches are powerful and versatile, they encounter difficulties when the moving load approaches the boundary of the domain and even beyond. These difficulties can be overcome by employing a large enough domain size but at the cost of significantly increased computational effort. In addition, the moving load is more frequently located at an intermediate location within an element other than the nodal points. This has been shown to lead to a reduction in the accuracy of the solution [7]. On the other hand, the moving element method (MEM) adopts a moving frameof-reference that travels with the moving load and thus the moving load is always located at a nodal point of one of the moving elements. Compared to the standard FEM, this numerical approach has been shown to be more accurate and efficient in dealing with various train-track dynamic problems [7-11].

In this paper, we present a computational study using the moving element method on the dynamic response of a high-speed train traveling on ballasted tracks accounting for hanging sleepers. The computational scheme presented in this paper will be first verified by comparison with available analytical results based on a simplified moving sprung mass model. Then a computational extended train-track model is put forward for the analysis of the dynamic response of the highspeed rail systems accounting for the effect of hanging sleepers. Parametric study will be conducted to investigate the effect of various factors affecting the dynamic response of the high-speed rail system. This 
includes the speed of the train, the number of hanging sleepers, and the number of fully supported sleepers that are surrounded by the hanging sleepers.

\section{Train-track model}

The problem of concern comprises a high-speed train traveling at speed $V$ over a ballasted track with or without hanging sleepers (see Fig. 1). The rail beam is resting over a number of equally spaced sleepers, which are again supported by the ballast. The ballast materials are idealized as discrete mass points that are interlocked with adjacent ballast points by their shear stiffness. These idealized ballast elements are supported by the subgrade above the rigid foundation. For sake of simplicity, only one rail beam of the railway track subjected to half weight of the train is considered.

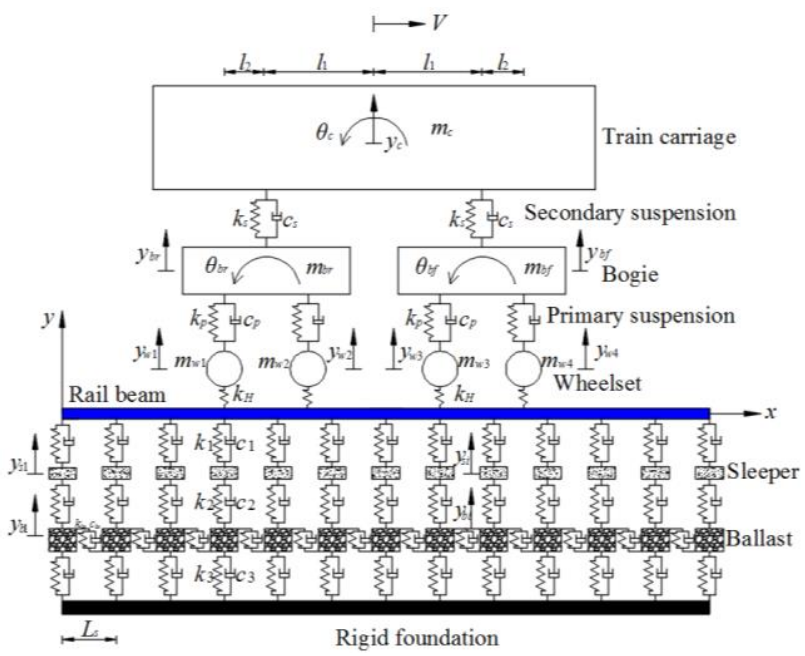

Fig. 1. Schematic drawing of high-speed train over ballasted track.

\subsection{Ballasted track model}

The ballasted track model comprises a rail beam that is supported by sleepers that have an equal spacing $L_{\mathrm{s}}$. The rail beam is modelled according to Timoshenko beam theory with density $\rho$, cross-sectional area $A$, area moment of inertia $I$, Young's modulus $E$, shear modulus $G$ and shear coefficient $\kappa$. The sleepers are modelled as rigid bodies with lumped mass $m_{s}$ and they are supported by the ballast elements with lumped mass $m_{b}$. The rail pads between the rail beam and the sleepers have stiffness coefficient $k_{1}$ and damping coefficient $c_{1}$. The ballast pavement supports the sleepers through stiffness $k_{2}$ and damping coefficient $c_{2}$. The governing equations of motion for the track model can be written as

$$
\begin{aligned}
& \rho A \frac{\partial^{2} y_{r}(x, t)}{\partial t^{2}}-\kappa A G \frac{\partial^{2} y_{r}(x, t)}{\partial x^{2}}+\kappa A G \frac{\partial \psi_{r}(x, t)}{\partial x} \\
& +\sum_{i=1}^{n} c_{1}\left(\frac{\partial y_{r}(x, t)}{\partial t}-\frac{\partial y_{s i}(t)}{\partial t}\right) \delta\left(x-i L_{s}\right) \\
& +\sum_{i=1}^{n} k_{1}\left(y_{r}(x, t)-y_{s i}(t)\right) \delta\left(x-i L_{s}\right)=-\sum_{j=1}^{m} F_{c j} \delta\left(x-X_{j}(t)\right)
\end{aligned}
$$

$$
\begin{aligned}
& \rho I \frac{\partial^{2} \psi_{r}(x, t)}{\partial t^{2}}-E I \frac{\partial^{2} \psi_{r}(x, t)}{\partial x^{2}}-\kappa A G \frac{\partial y_{r}(x, t)}{\partial x} \\
& +\kappa A G \psi_{r}(x, t)=0 \\
& m_{s} \frac{\partial^{2} y_{s i}(t)}{\partial t^{2}}+c_{1}\left(\frac{\partial y_{s i}(t)}{\partial t}-\left.\frac{\partial y_{r}(x, t)}{\partial t}\right|_{x=(i-1) L_{s}}\right)+c_{2}\left(\frac{\partial y_{s i}(x, t)}{\partial t}-\frac{\partial y_{b i}(x, t)}{\partial t}\right) \\
& +k_{1}\left(y_{s i}(t)-\left.y_{r}(x, t)\right|_{x=(i-1) L_{s}}\right)+k_{2}\left(y_{s i}(t)-y_{b i}(t)\right)=0 \\
& m_{b} \frac{\partial^{2} y_{b i}(t)}{\partial t^{2}}+c_{2}\left(\frac{\partial y_{b i}(t)}{\partial t}-\frac{\partial y_{s i}(t)}{\partial t}\right)+c_{3} \frac{\partial y_{b i}(x, t)}{\partial t} \\
& +c_{b s}\left(2 \frac{\partial y_{b i}(t)}{\partial t}-\frac{\partial y_{b i-1}(t)}{\partial t}-\frac{\partial y_{b i+1}(t)}{\partial t}\right)+k_{2}\left(y_{b i}(t)-y_{s i}(t)\right) \\
& +k_{3} y_{b i}(t)+k_{b s}\left(2 y_{b i}(t)-y_{b i-1}(t)-y_{b i+1}(t)\right)=0
\end{aligned}
$$

where $y_{r}$ denotes the displacement of the rail beam; $y_{s i}$ the displacement of the $i$ th sleeper; $\psi_{r}$ the bending rotation of the rail; $k_{1}$ and $c_{1}$ the stiffness and damping coefficient of the rail pad; $k_{2}$ and $c_{2}$ the stiffness and damping coefficients of the ballast; $k_{3}$ and $c_{3}$ are the stiffness and damping coefficients of the subgrade. $k_{b s}$ and $c_{b s}$ are the shear stiffness and damping coefficient between two discrete ballast elements. $F_{c j}$ is the contact force between the $j$ th train wheel and the rail beam; $X_{j}$ is the travel distance of the $j$ th wheel; and $\delta$ the Dirac-delta function.

\subsection{Train model}

The train is modelled as a 10-DOFs (degrees of freedom) sprung-mass system comprising one train body, two bogies and four wheelsets. These train components are modelled as rigid bodies interconnected by suspension systems modelled as spring-dashpot units. The vehicle carriage has a lumped mass $\mathrm{m}_{\mathrm{c}}$ and moment of inertia $I_{c}$. The two bogies have an identical mass $m_{b g}$ and moment of inertia $I_{b}$. Each of the four wheelsets has a mass $m_{w}$. Based on Newton's second law of motion, the governing equations of the moving sprung-mass train model can be written in compact form as

$$
\mathbf{M}_{v} \ddot{\mathbf{y}}_{v}+\mathbf{C}_{v} \dot{\mathbf{y}}_{v}+\mathbf{K}_{v} \mathbf{y}_{v}=\mathbf{P}_{v}
$$

where $\mathbf{M}_{v}, \mathbf{C}_{v}, \mathbf{K}_{v}$ and $\mathbf{P}_{v}$ denote the mass, damping, stiffness matrices and force vector of the vehicle model; $\mathbf{y}_{v}$ is the vector of train degrees of freedom and is given as

$$
\mathbf{y}_{v}=\left\{\begin{array}{llllllllll}
y_{c} & \theta_{c} & y_{b r} & \theta_{b r} & y_{b f} & \theta_{b f} & y_{w 1} & y_{w 2} & y_{w 3} & y_{w 4}
\end{array}\right\}^{\mathrm{T}}
$$

herein $y_{c}$ and $\theta_{c}$ denote the vertical displacement and pitching rotation of the carriage, respectively; $y_{b r}, \theta_{b r}$ and $y_{b f}, \theta_{b r}$ are the vertical displacement and pitching rotation of the bogie at the rear and the one in the front of the vehicle, respectively; $y_{w i}$ is the vertical displacement of the $i$ th wheelset.

\subsection{Wheel and rail interaction}

The vehicle model and the track model are coupled together by means of wheel and rail interaction. Here in 
this paper, the nonlinear Hertz contact model is adopted by assuming that the contact area is always circular [68]. This contact model also allows the possible separation between the wheel and the rail known as the "jumping wheel phenomenon".

\section{Moving element method}

The moving element method (MEM) is employed here for the dynamic analysis of the train-track system in the time domain. This numerical method employs a moving $r$-axis whose origin is attached to the train and travels with it at the same speed $V$ and requires the governing equations of motion to be reformulated in the moving frame-of-reference. For the analysis of discretely supported high-speed rail (HSR), the efficient computational scheme recently proposed by Dai et al. [11] which pre-prepares the global stiffness and damping matrices in one period for easy retrieval in the subsequent time domain computation can be adopted. In addition, the movement of the hanging sleeper(s) must be considered. The computation can thus be divided into three phases. In the first phase, the truncated track segment has not reached the location of the hanging sleeper, as shown in Fig. 2. The computation in this phase thus does not involve the unsupported sleeper. In the second phase, the truncated track has partially travelled across the unsupported sleeper. During this phase, the stiffness and damping matrices of the ballasted track system must be updated at each time step to account for the change due to the loss of ballast support under the incident sleeper. In the third phase, the truncated track has completely travelled past the unsupported sleeper and is now supported fully by wellbedded sleepers. The computation in this phase is identical to that in the first phase.

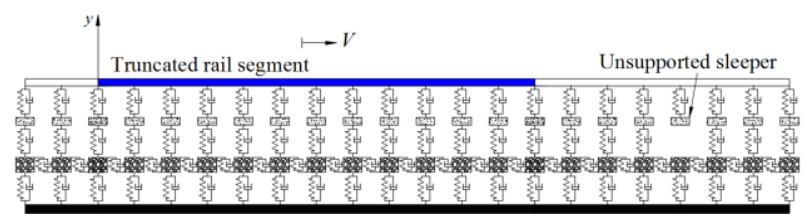

Fig. 2. Truncated track in vicinity of unsupported sleeper.

\section{Numerical study}

Two cases involving hanging sleepers are investigated in this paper. The first case involves a simplified discretely supported rail beam subject to a moving mass. The aim of this case is to examine the accuracy of the proposed numerical model through comparison with available analytical results in the literature. In the second case, the full model comprising the 10-DOF train model and 3layer ballasted track model is employed to investigate the dynamic response of a HSR system accounting for one or more hanging sleepers with different patterns.

\subsection{Comparison of results}

In an attempt to examine the accuracy of the methodology presented in Section 3, the case of a simplified discretely supported track subjected to a moving sprung mass as studied by Sheng et al. [12] is first considered. The model of concern is shown in Fig. 3. The rail beam is represented by a Timoshenko beam resting on evenly spaced spring-dashpot units. The wheel load is modelled as a sprung-mass traveling at $80 \mathrm{~m} / \mathrm{s}$. Table 1 lists the parameters for the analysis model adopted in [12]. Note that the railhead roughness is represented by a sine function with amplitude $a_{t}=10 \times$ $10^{-6} \mathrm{~m}$ and wavelength $\lambda_{t}=0.04 \mathrm{~m}$. In view of the speed, the frequency of the excitation due to the railhead roughness is $2000 \mathrm{~Hz}$.

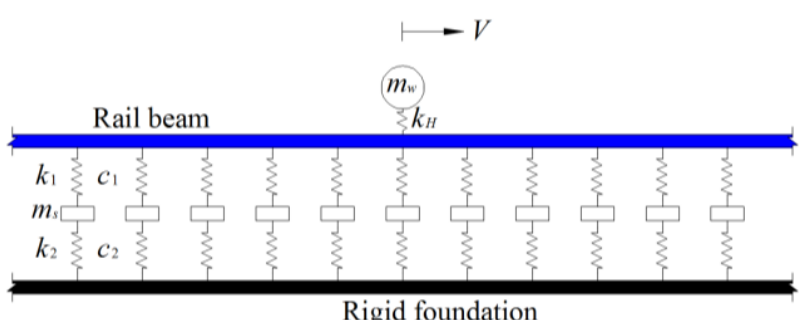

Fig. 3. Simplified model for numerical verification.

Table 1. Parameters for the simplified train-track model.

\begin{tabular}{|c|c|c|c|}
\hline Parameter & Value & Parameter & Value \\
\hline$\rho A$ & $60.3665 \mathrm{~kg} / \mathrm{m}$ & $\kappa$ & 0.4 \\
\hline$E I$ & $\begin{array}{c}6.4155 \times 10^{6} \\
\mathrm{Nm}^{2}\end{array}$ & $m_{w}$ & $1350 \mathrm{~kg}$ \\
\hline$G A$ & $\begin{array}{c}6.2289 \times 10^{9} \\
\mathrm{~N}\end{array}$ & $m_{s}$ & $162 \mathrm{~kg}$ \\
\hline$L_{s}$ & $0.6 \mathrm{~m}$ & $k_{H}$ & $\begin{array}{c}1.4 \times 10^{9} \\
\mathrm{~N} / \mathrm{m}\end{array}$ \\
\hline$k_{1}$ & $3.5 \times 10^{8} \mathrm{~N} / \mathrm{m}$ & $k_{2}$ & $\begin{array}{c}50 \times 10^{6} \\
\mathrm{~N} / \mathrm{m}\end{array}$ \\
\hline
\end{tabular}

In the MEM model, a rail beam segment of length 18 $\mathrm{m}$ is truncated. This segment covers 30 sleeper bays and based on a convergence study, it is found that the length is long enough to avoid the boundary effect in simulating the behaviour of an infinitely long rail. The beam element size is set to $0.3 \mathrm{~m}$. This means the track segment between two adjacent sleepers is discretized into two elements only. In order to capture the dynamic response due to the high-frequency excitation, the time step size is set to $7.5 \times 10^{-6} \mathrm{~s}$. This is equivalent to 100 steps for the sprung mass to travel across one entire sleeper bay.

Figure 4 shows the steady-state dynamic wheel-rail contact force predicted by the MEM for the case when the sprung mass travels from one sleeper to the next. Also presented in this figure are the results published by Sheng et al. [12]. As can be seen, both results match well with each other, thereby validating present 
computational approach for the analysis of discrete ballasted tracks under moving loads.

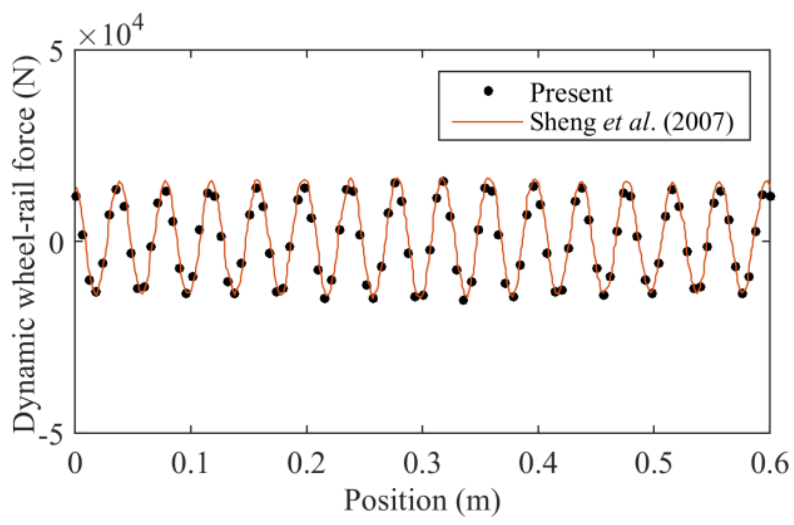

Fig. 4. Dynamic contact force over one sleeper bay.

\subsection{Effect of hanging sleepers}

The dynamic response of a high-speed rail system accounting for hanging sleepers is next investigated. In this study, the train-track coupled model described in Section 2 is employed for the analysis. The parameters for the train model are listed in Table 2. Table 3 lists the parameters for the track model where the rail beam model is based on the Chinese type 60 rail. The nonlinear wheel-rail contact constant is $1 / k_{H}=4.27 \times 10^{-8}$ $\mathrm{m} / \mathrm{N}^{2 / 3}$. Note that all the parameters listed above are reported by Zhu et al. [4]. Note that smooth railhead is purposely chosen such that the effect unsupported sleepers will not be overweighed by the existence of track irregularities.

Table 2. Parameters for the train model.

\begin{tabular}{|c|c|c|c|}
\hline Parameter & Value & Parameter & Value \\
\hline$m_{c}$ & $26000 \mathrm{~kg}$ & $m_{b f}$ & $2309 \mathrm{~kg}$ \\
\hline$m_{b r}$ & $2309 \mathrm{~kg}$ & $m_{w}$ & $750 \mathrm{~kg}$ \\
\hline$I_{c}$ & $\begin{array}{c}1.155 \times 10^{6} \\
\mathrm{kgm}^{2}\end{array}$ & $I_{b}$ & $1970 \mathrm{kgm}^{2}$ \\
\hline$k_{p}$ & $1.87 \mathrm{MN} / \mathrm{m}$ & $c_{p}$ & $5 \times 10^{5} \mathrm{Ns} / \mathrm{m}$ \\
\hline$k_{s}$ & $1.72 \mathrm{MN} / \mathrm{m}$ & $c_{s}$ & $\begin{array}{c}1.96 \times 10^{5} \\
\mathrm{Ns} / \mathrm{m}\end{array}$ \\
\hline$l_{1}$ & $9 \mathrm{~m}$ & $l_{2}$ & $1.25 \mathrm{~m}$ \\
\hline
\end{tabular}

In this study, various cases considering different patterns of hanging sleepers are investigated. Figure 5 shows 6 patterns. The first case involves only one hanging sleeper. The rest cases involve two hanging sleepers spaced apart by different numbers of fully supported sleepers. In view of this, the first case can also be regarded as the case involving two hanging sleepers that are spaced apart by an infinite number of fully supported sleepers.
Table 3. Parameters for the track model.

\begin{tabular}{|c|c|c|c|}
\hline Parameter & Value & Parameter & Value \\
\hline$\rho A$ & $60.64 \mathrm{~kg} / \mathrm{m}$ & $\kappa$ & 0.4 \\
\hline$E I$ & $\begin{array}{c}6.7557 \times 10^{6} \\
\mathrm{Nm}^{2}\end{array}$ & $v$ & 0.3 \\
\hline$m_{s}$ & $130 \mathrm{~kg}$ & $m_{b}$ & $460 \mathrm{~kg}$ \\
\hline$k_{1}$ & $120 \mathrm{MN} / \mathrm{m}$ & $c_{1}$ & $\begin{array}{l}7.5 \times 10^{4} \\
\mathrm{Ns} / \mathrm{m}\end{array}$ \\
\hline$k_{2}$ & $180 \mathrm{MN} / \mathrm{m}$ & $c_{2}$ & $\begin{array}{c}5.88 \times 10^{4} \\
\mathrm{Ns} / \mathrm{m}\end{array}$ \\
\hline$k_{3}$ & $120 \mathrm{MN} / \mathrm{m}$ & $c_{3}$ & $\begin{array}{c}3.12 \times 10^{4} \\
\mathrm{Ns} / \mathrm{m}\end{array}$ \\
\hline$k_{b s}$ & $78 \mathrm{MN} / \mathrm{s}$ & $c_{b s}$ & $8 \times 10^{4} \mathrm{Ns} / \mathrm{m}$ \\
\hline
\end{tabular}
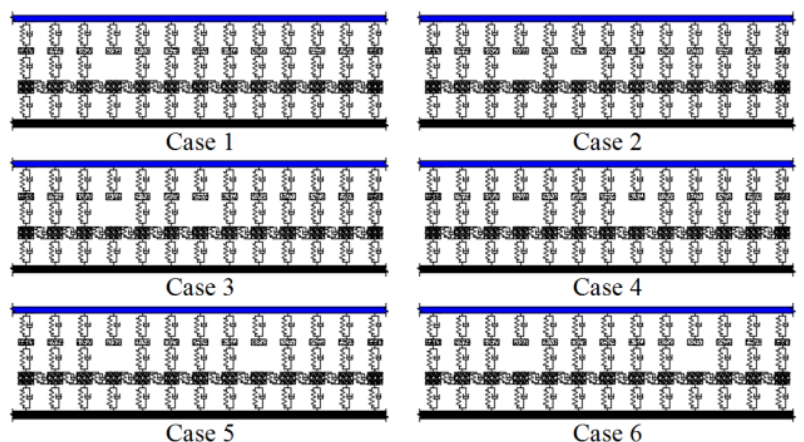

Fig. 5. 6 different patterns of hanging sleepers.

Figure 6 shows the wheel-rail contact forces in the vicinity of the hanging sleepers for different cases when the speed of the train is $320 \mathrm{~km} / \mathrm{h}$. As can be seen, the maximum contact force in the second case is smaller than the first case by about $9 \%$, despite of the increase in the total number of hanging sleepers. This finding coincides with the results reported in [1]. However, as the number of fully supported sleepers between two hanging sleepers increases from case 3 to case 6 , it is interesting to find that the maximum contact force increases to a peak of $122.9 \mathrm{kN}$ in case 4 before decreases. As the number of fully supported sleepers increases to 5 , the maximum contact force reduces to the same value as in the base case 1 . Further study with more fully supported sleepers between two hanging sleepers revealed that the maximum contact force remains unchanged. In other words, the effect of the first hanging sleeper has been fully damped out before the wheelsets reach the second hanging sleeper for cases when the number of fully supported sleepers between the faulty exceeds 4 .

Figure 7 presents the maximum DAF in wheel-rail contact force for case 1 to case 9 . Note that cases 7, 8 and 9 refer to the patterns where two hanging sleepers are spaced apart by 6,7 and 8 fully supported sleepers, respectively. As can be seen, case 2 tends to give the lowest maximum contact forces for all cruising speeds of the train considered in this study. The highest maximum 
contact forces are found to occur for cases 3-5, depending on the speed of the train. For smaller cruising speeds, the highest maximum contact force tends to occur for the case where the space between two hanging sleepers is narrower as compared to higher speeds. This may be explained as due to the phase difference in the dynamic response to the excitation, the maximum response is expected to occur slightly after the excitation. For higher speeds, the maximum contact force is expected to occur at longer distances after traveling across the unsupported sleeper. As the distance between the two hanging sleepers further increases, it is found that maximum contact forces reduce to those observed in case 1. This implies that these two hanging sleepers have independent effects on the dynamic response of the highspeed rail system when they are spaced far apart.

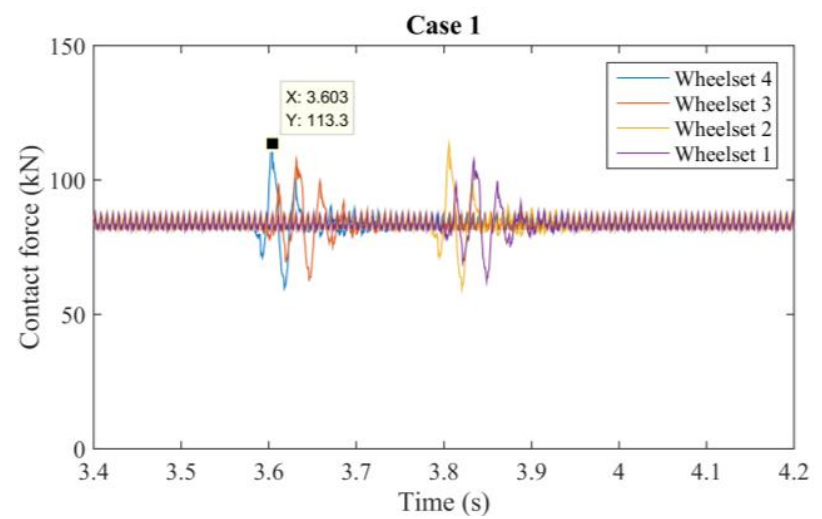

(a)

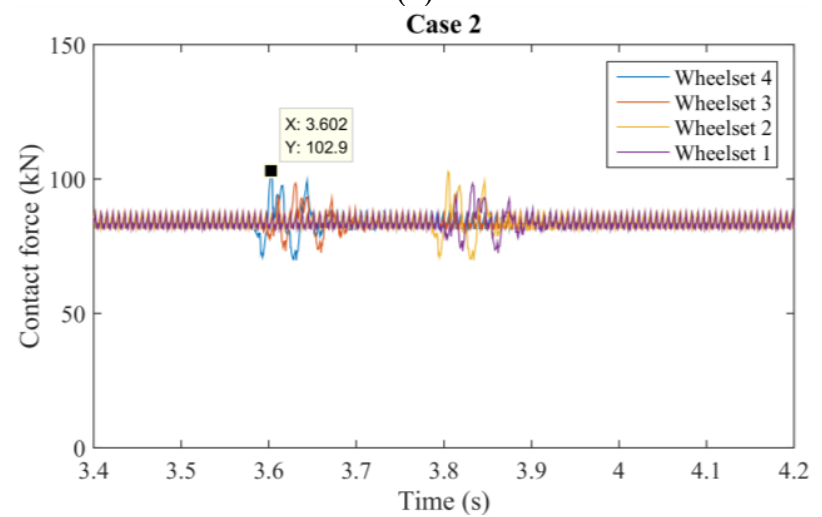

(b)

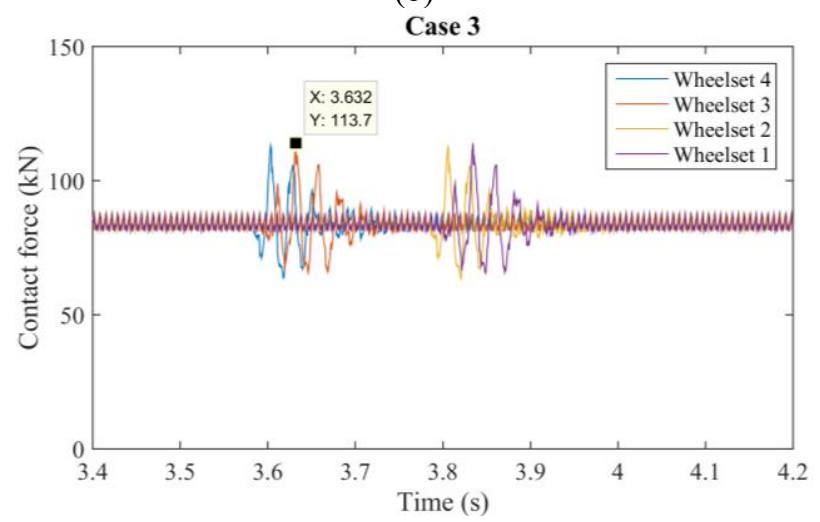

(c)

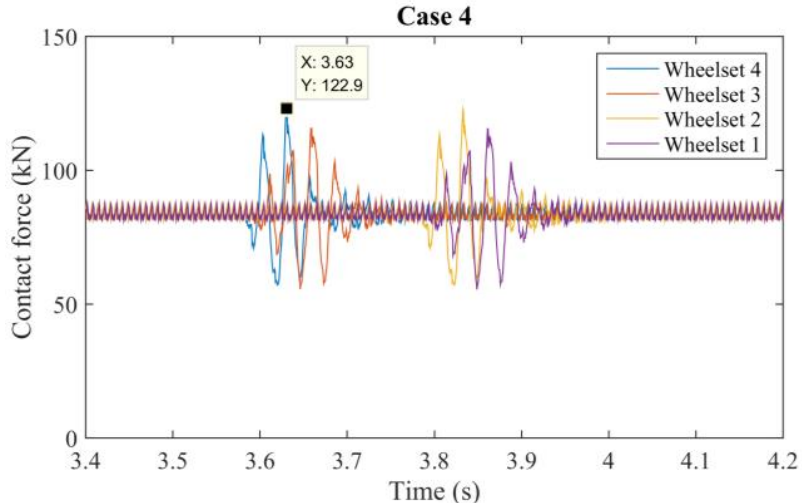

(d)

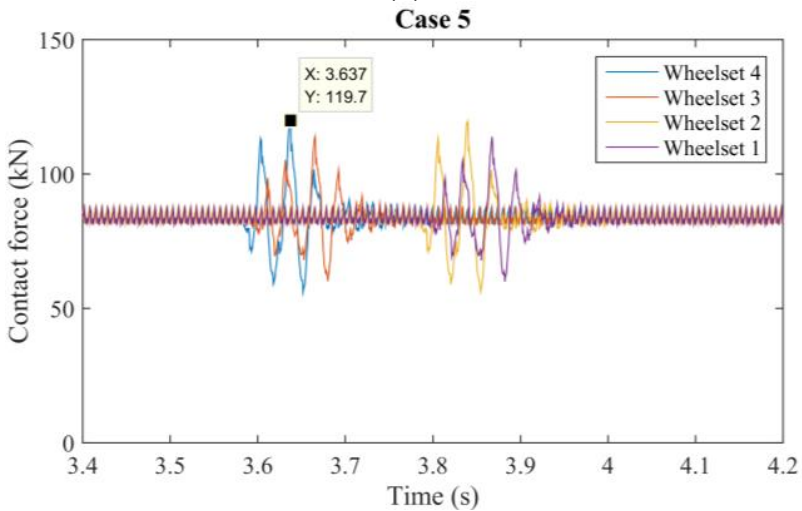

(e)

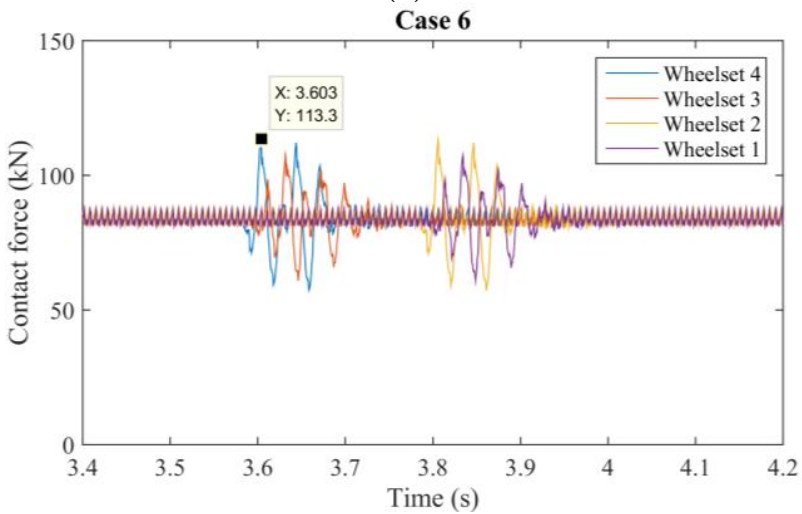

(f)

Fig. 6. Wheel-rail contact forces for 6 cases.

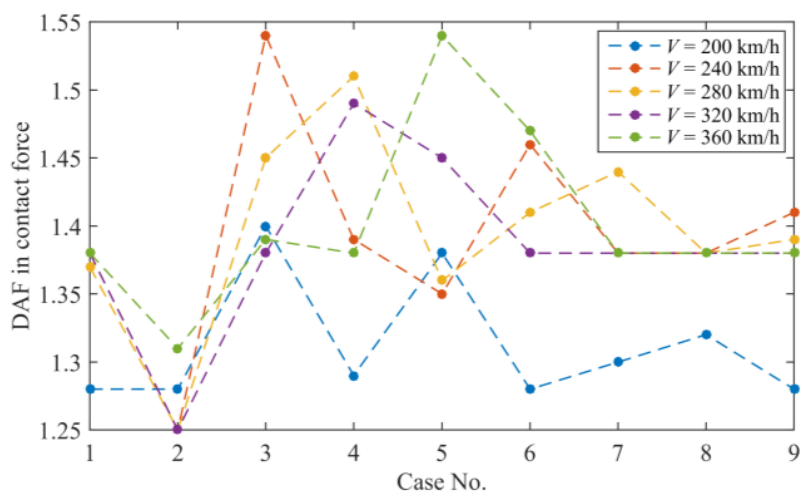

Fig. 7. DAF in contact force for different cases. 


\section{Conclusion}

This paper is concerned with a computational study on the dynamic responses of a high-speed rail system accounting for hanging sleepers. A computational scheme in conjunction with the moving element method is proposed to account for the motion of the rail substructures and the unsupported sleepers with respect to the truncated rail segment. The accuracy of the proposed method is examined by comparison with available analytical results using a simplified moving sprung mass model. A good agreement is found between the present solution and the available results in the literature, thereby validating the proposed computational scheme for the analysis of ballasted track under moving loads.

In the analysis of high-speed rail systems accounting for hanging sleepers using an extended 10-degree-offreedom moving sprung mass model, it is found that the speed of the train and the pattern of the hanging sleepers are two key factors affecting the dynamic response of the system. Generally, the lowest dynamic response in terms of wheel-rail contact force is expected to occur for the case of one fully supported sleeper surrounded by two hanging sleepers for all speeds considered in this study. The highest maximum contact force is observed to occur when the two hanging sleepers are spaced apart by 3 to 5 fully supported sleepers. This implies that the maintenance of the track must be conducted carefully to avoid the most undesirable situation. As the spacing between the two hanging sleepers further increases to 7 sleeper bays and beyond, the coupled effect of the two hanging sleepers becomes small and may be neglected.

\section{References}

1. A. Lundqvist, T. Dahlberg, Proc. IMechE. Part F: J. Rail Rapid Transit 219, 67-77 (2005)

2. S. Augustin, G. Gudehus, G. Huber, A. Schunemann, System dynamics and long-term behaviour of railway vehicles, track and subgrade, 6, 317-336 (Springer, Berlin, 2003)

3. J.J. Zhu, A.K.W. Ahmed, S. Rakheja, A. Khajepour, Vehicle Syst. Dyn., 48, 1535-1552 (2010)

4. J.Y. Zhu, D.J. Thompson, C.J.C. Jones, Vehicle Syst. Dyn., 49, 1389-1408 (2011)

5. J.A. Zakeri, M. Fattahi, M.M. Ghanimoghadam, J. Mech. Sci. Technol., 29, 2289-2295 (2015)

6. C. Esveld, Modern railway track (MRT Productions, Duisburg, 2001)

7. K.K. Ang, J. Dai, J. Sound Vib., 332, 2954-2970 (2013)

8. K.K. Ang, J. Dai, M.T. Tran, V.H. Luong, Int. J. Comp. Meth., 11, 1-12 (2014)

9. C.G. Koh, J.S.Y. Ong, D.K.H. Chua, J. Feng, Int. J. Numer. Meth. Eng., 56, 1549-1567 (2003)

10. M.T. Tran, K.K. Ang, V.H. Luong, J. Sound Vib., 333, 5427-5442 (2014)
11. J. Dai, K.K. Ang, M.T. Tran, V.H. Luong, D. Jiang, Proc. IMechE. Part F: J. Rail Rapid Transit (2017)

12. X. Sheng, M. Li, C.J.C. Jones, D.J. Thompson, J. Sound Vib. 303, 873-894 (2007) 